\title{
No differences in knee kinematics between active and passive flexion-extension movement: an intra-operative kinematic analysis performed during total knee arthroplasty
}

\author{
Alberto Grassi ${ }^{1}$, Nicola Pizza ${ }^{*}$, Nicola Francesco Lopomo ${ }^{3}$, Maurilio Marcacci ${ }^{4}$, Michele Capozzi ${ }^{5}$, \\ Giulio Maria Marcheggiani Muccioli ${ }^{1}$, Francesca Colle ${ }^{5}$ and Stefano Zaffagnini ${ }^{1,2}$
}

\begin{abstract}
Purpose: The objective of the present study was to acquire and compare by the use of a navigation system the intra-operative flexion-extension movement of the knee performed actively by the patient and passively by the surgeon before and after a total knee arthroplasty (TKA) implantation.

Methods: A cohort of 31 patients with primary knee osteoarthritis (OA), candidate for TKA underwent intraoperative kinematics assessment with a commercial navigation system before and after the definitive implant positioning of a Cruciate Retaining (CR) Mobile Bearing (MB) prostheses. The kinematical data were acquired while surgeon performed the flexion-extension movement (passive ROM - pROM), and while the patient performed it (active ROM - aROM). Differences between pre- and post- implantation and between active and passive motions, were statistically analyzed using paired Student t-tests $(p=0.05)$.

Results: No statistically significant difference were found between aROM and pROM with paired Student t-test regarding internal-external rotation and anterior-posterior translation of the femoral component with respect to the tibia during flexion-extension movement before and after TKA implant $(p>0.05)$.

Conclusions: Active muscle contraction seems to not significantly affect TKA kinematics. The ROM performed by the surgeon during operation resemble the movement actively performed by the patient.

The clinical relevance of this study further supports the use of CAS system in performing intra-operative analysis concerning knee biomechanics.
\end{abstract}

Keywords: Total knee Arthroplasty, Total knee replacement, Active and passive flexion, Kinematics, Navigation system

\footnotetext{
* Correspondence: nicola.pizza@ior.it

${ }^{1}$ |l Clinica Ortopedica e Traumatologica, IRCCS, Istituto Ortopedico Rizzoli,

Bologna, Italy

Full list of author information is available at the end of the article
}

\section{Springer Open}

(c) The Author(s). 2020 Open Access This article is licensed under a Creative Commons Attribution 4.0 International License, which permits use, sharing, adaptation, distribution and reproduction in any medium or format, as long as you give appropriate credit to the original author(s) and the source, provide a link to the Creative Commons licence, and indicate if changes were made. The images or other third party material in this article are included in the article's Creative Commons licence, unless indicated otherwise in a credit line to the material. If material is not included in the article's Creative Commons licence and your intended use is not permitted by statutory regulation or exceeds the permitted use, you will need to obtain permission directly from the copyright holder. To view a copy of this licence, visit http://creativecommons.org/licenses/by/4.0/. 


\section{Background}

The most recent uses of Computer-Assisted-Surgery (CAS) in Total Knee Arthroplasty (TKA) provide the possibility to intra-operatively assess the functional behavior of the knee joint [7-11]. In particular, CAS is able to estimate range of motion and laxities associated with the patient and joint-specific surgical reconstruction. The real-time intra-operative kinematic assessment allows the comparison between the pathological condition (i.e. before the reconstruction) and the newly restored condition (i.e. after the reconstruction). One of the major issues related to the navigation systems concerns its capacity to evaluate only the passive kinematics, hence without taking into account the muscular control of the lower limb. Doro et al. [6], in fact, criticize the lack of active muscular contraction by the patient during the intra-operative evaluation, thus stating that this technology is limited for a proper biomechanical assessment of the knee joint.

Hence, the purpose of the present study was to acquire and compare the intra-operative flexion-extension movement of the knee performed actively by the patient and passively by the surgeon before and after the implantation of a TKA.

The hypothesis was that active knee flexion-extension movement would show comparable pattern to the passive one, demonstrating that even passive kinematics can properly describe the biomechanical behavior of the knee joint.

The clinical relevance of the present study derives from the possibility of evaluating the effects of patient's active muscular contraction on the kinematics of the osteoarthritic knee and of the TKA hence to better understand the movement of this joint in its complexity.

\section{Materials and methods}

\section{Patients selection}

The study was approved by the ethical committee of the IRCCS Rizzoli Orthopedic Institute (protocol number 11551/CE/US/ml, 5 May 2006).

A cohort of 31 patients with knee osteoarthritis (OA) candidate for TKA was enrolled for the present study after signing an informed consent between 2011 and 2012.

The inclusion criteria were: (1) Primary knee osteoarthritis, (2) Kellgren-Lawrence grade 3-4, (3) BMI < 40 $\mathrm{kg} / \mathrm{m} 2$. The exclusion criteria were: (1) Previous lower limb alignment corrective surgery on the affected side, (2) BMI $>40 \mathrm{~kg} / \mathrm{m} 2$, (3) Rheumatoid arthritis, (4) Posttraumatic arthritis.

The mean age of the patients included in the study was $70.5 \pm 6.5$ years (range $83-54$ years), 9 males and 22 females.

\section{Acquisition protocol}

All the patients underwent intra-operative kinematics assessment with a commercial navigation system (BLUIGS Orthokey, Lewes, Delaware) equipped with a software specifically focused on kinematic analysis (KLEE, Orthokey, Lewes, Delaware) [14]. This system has a 3D RMS volumetric accuracy of $0.350 \mathrm{~mm}$ and a 3D RMS volumetric repeatability of $0.200 \mathrm{~mm}$ [21], as reported by the producer. All the kinematic data were off-line processed by applying proprietary routines developed in Matlab (Mathworks, Natick, MA, USA).

The proposed methodology was assessed to have a repeatability lower than $2 \mathrm{~mm}$ in translation and lower than $3^{\circ}$ in rotations [14], with ICC values ranging from 0.94 to 0.99 [4].

Anatomical landmarks on femur and tibia were acquired to define the joint coordinate reference system (JCS) $[5,8]$ and to perform TKA navigation protocol. The anatomical registration on the femur consisted of: the femoral head (by leg pivoting), the most distal part of the femur in the intercondylar notch (over to the lateral margin of the posterior cruciate ligament), the anterior shaft, the medial and lateral epicondyles, the most posterior and distal part of the condyles and the Whiteside Line (WSL). The medial and lateral malleoli, the tibial spine, the tibial tuberosity and the lateral and medial plateaus were acquired on the tibia.

Using the anatomical landmarks, the navigation system was able to automatically identify the femoral mechanical axis, surgical trans-epicondylar axis (TEA), WSL, posterior condylar line for femur and tibial mechanical axis and the line connecting medial and lateral tibial plateau for tibia (Fig. 1).

For each patient, intra-operative kinematic acquisitions were collected before and after the definitive implant positioning. Pre-operative kinematic tests were specifically acquired after skin incision to allow the fixation of the tibial and femoral trackers, after medial parapatellar arthrotomy, before patella luxation and meniscal and anterior cruciate ligament (ACL) removal, while post-implant kinematic acquisitions were collected after the cementation of definitive prosthesis. Both the pre-operative and the post-operative acquisition were acquired with the tourniquet inflated, the joint capsule open and patella reduced. The kinematical data were acquired performing flexion-extension movements (full extension-full flexion-full extension), three times for each subject in two different conditions (Fig. 2): the passive motion (pROM), manually performed by the surgeon, maintaining the foot in neutral position (i.e. not introducing any additional stress/torque at foot level during the flexion-extension movement), and the active movement (aROM), directly performed by the patient. 


\section{FEMUR}

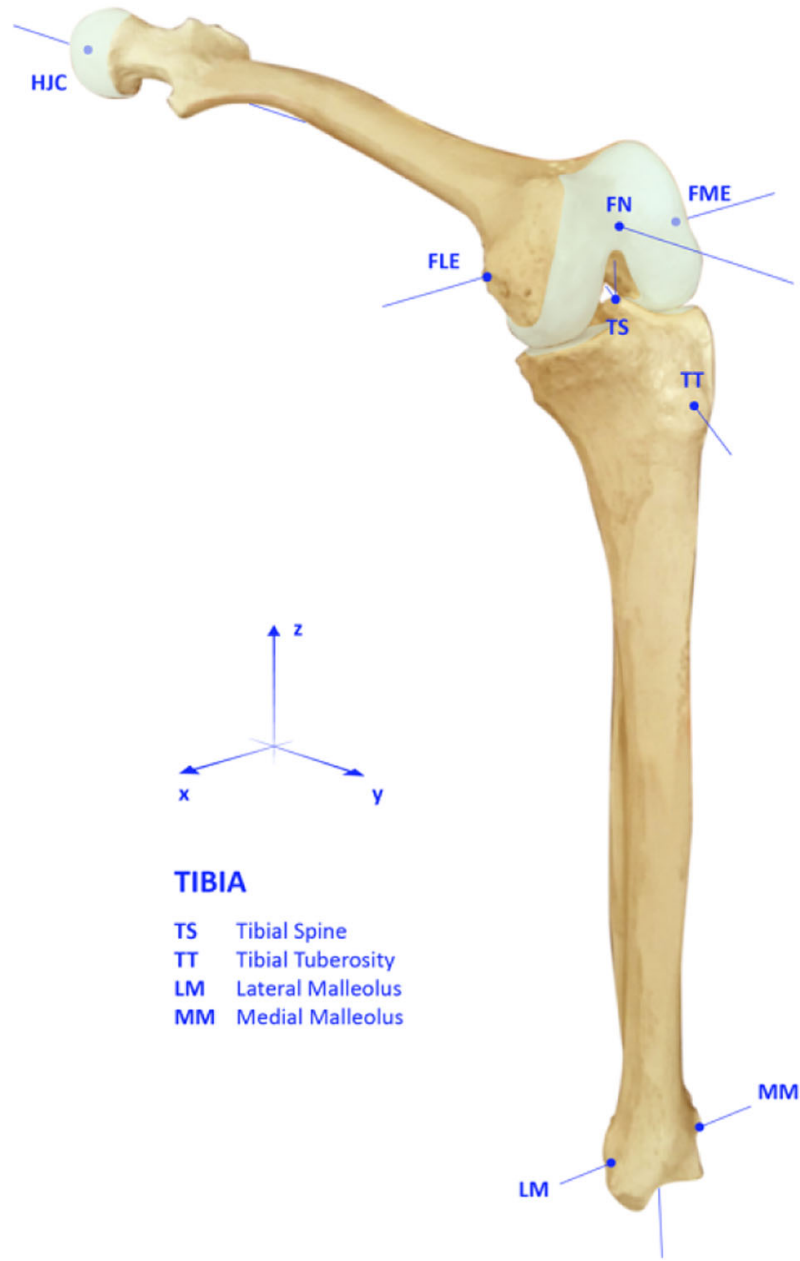

HJC Hip Joint Center

FN Femoral Notch

FLE Femoral Lateral Epicondyle

FME Femoral Medial Epicondyle

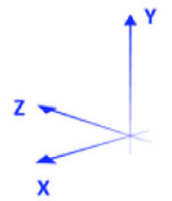

Fig. 1 Anatomical reference systems used for the kinematic analysis

\section{Surgical technique}

All the surgeries were performed under combined spinal and epidural anesthesia (CSE technique) which is a wellknown technique typically used during labor. It offers the benefits of rapid onset of analgesia and at the same time allows lower-limb motor power [17]. Therefore, with the use of CSE anesthesia, the patients were able to perform active knee flexion-extension movement after skin incision and tracker positioning for navigation without experiencing pain. A midline skin incision was performed, and both femoral and tibial tracker were positioned in order to not interfere with surgical technique and prevent accidental mobilization. A standard medial parapatellar arthrotomy was performed and the patella was everted. Menisci and ACL were resected, and a tibial cut was made sparing PCL. After the cut of distal femur, the 4-in-1 guide was used to complete the femoral cut with the opportune size. The trial components were positioned and the flexion-extension gaps opportunely balanced, when needed. After the patellar cut and pulsed washing, definitive prothesis was implanted and the tourniquet finally released.

All patients were operated with the standard technique (medial parapatellar approach, adjusted mechanical alignment) and received a cemented Cruciate Retaining (CR) highly congruent Mobile Bearing (MB) TKA (Gemini, Waldemar LINK GmbH \& Co. KG, Barkhausenweg 10, 22,339 Hamburg, Germany) with patella resurfacing.

\section{Data analysis}

The coordinate reference system on femur was defined as follows: the femoral mechanical axis as the proximaldistal (PD) axis, the anterior-posterior (AP) axis as the cross product between the $\mathrm{PD}$-axis and the surgical TEA, and the cross product between AP-axis and PDaxis as the medial-lateral (ML) axis, thus achieving an anatomic orthogonal reference system. 

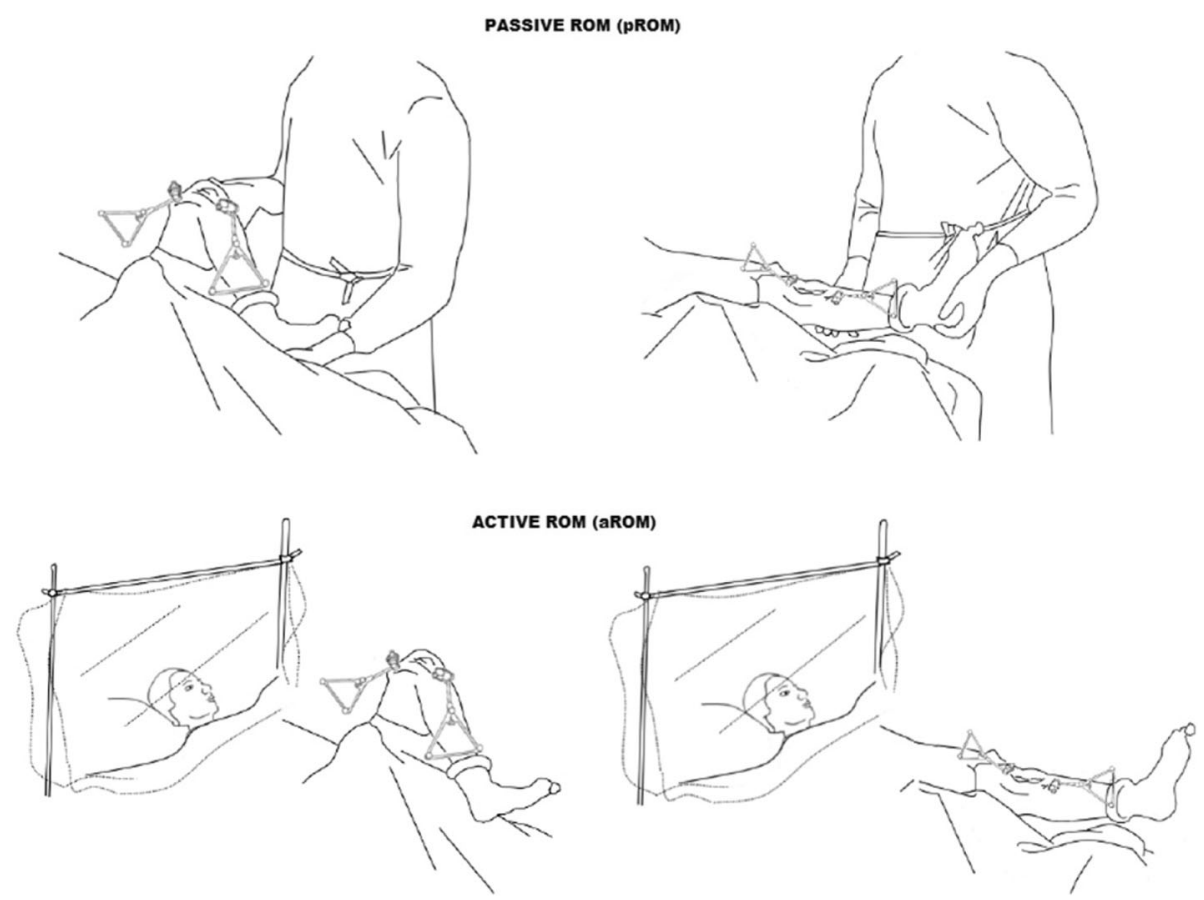

Fig. 2 pROM: Passive flexion-extension movement performed by the surgeon (A). aROM: Active flexion-extension movement performed by the patient during surgery in CSE anesthesia

The anatomic orthogonal reference system of the tibia was defined as: the PD-axis set as the tibial mechanical axis, the ML-axis as the cross product between the line connecting tibial spine and tibial tuberosity and the PDaxis, and the AP-axis as the cross-product between PDaxis and ML-axis.

Based on the acquired flexion-extension movements, the internal-external (IE) rotations were plotted against knee flexion. The AP translation was computed for both the medial and lateral epicondyles, evaluating their displacement projected in the transverse plane on the tibial reference system.

\section{Statistical analysis}

Starting from the analysis of literature [1, 2], a priori power analysis for a two-tailed paired Student's t-test (alfa $=0.05$, power $=0.8$, mean difference of $3.0 \pm 5.0^{\circ}$ of rotations and $3.0 \pm 5.0 \mathrm{~mm}$ of displacements) indicated a minimum sample size of 24 subjects.

For statistical comparison of the kinematic behavior, continuous data obtained from passive and active movements from $0^{\circ}$ to $120^{\circ}$, both in pre- and post-operative conditions, were re-sampled each $5^{\circ}$ of knee flexion using a smooth curve-fitting function that enabled direct comparison of patient.

IE rotations and AP translations values were then averaged on the three repetitions, at every re-sampled angle. The mean values obtained for each subject were then averaged for the whole cohort, thus obtaining one mean curve for the active condition and one for the passive one.

Both in pre- and post-implant conditions, internalexternal (IE) rotations and anterior-posterior (AP) translations were estimated for pROM and aROM kinematic tests.

Differences between pre- and post- implantation and between active and passive motions, were statistically analysed using paired Student t-tests $(p=0.05)$. Statistical significance was set at 95\% $(\mathrm{p}=0.05)$. Analyse-it software (Analyse-it Software, Ltd., The Tannery 91 Kirkstall Road, Leeds, LS3 1HS, United Kingdom) was used to perform the reported statistical analysis.

\section{Results}

\section{Tibial IE rotation during flexion}

Pre-operative rotation patterns were comparable between active and passive motions, despite aROM showed slightly larger values of internal tibial rotations (Fig. 3).

In fact, in full extension ( $0^{\circ}$ of flexion) aROM showed an average external tibial rotation of $9.5^{\circ} \pm 7.9^{\circ}$ while pROM showed an average external tibial rotation of $2.5^{\circ} \pm 11.2^{\circ}$. In early flexion $\left(0^{\circ}-30^{\circ}\right)$, the screw-home mechanism was observed in both conditions, with an internal tibial rotation of about $9^{\circ}$ for active movements and $6^{\circ}$ for passive movements. Beyond $30^{\circ}$ and up to $120^{\circ}$, tibial rotation showed a further gradual decrease in 


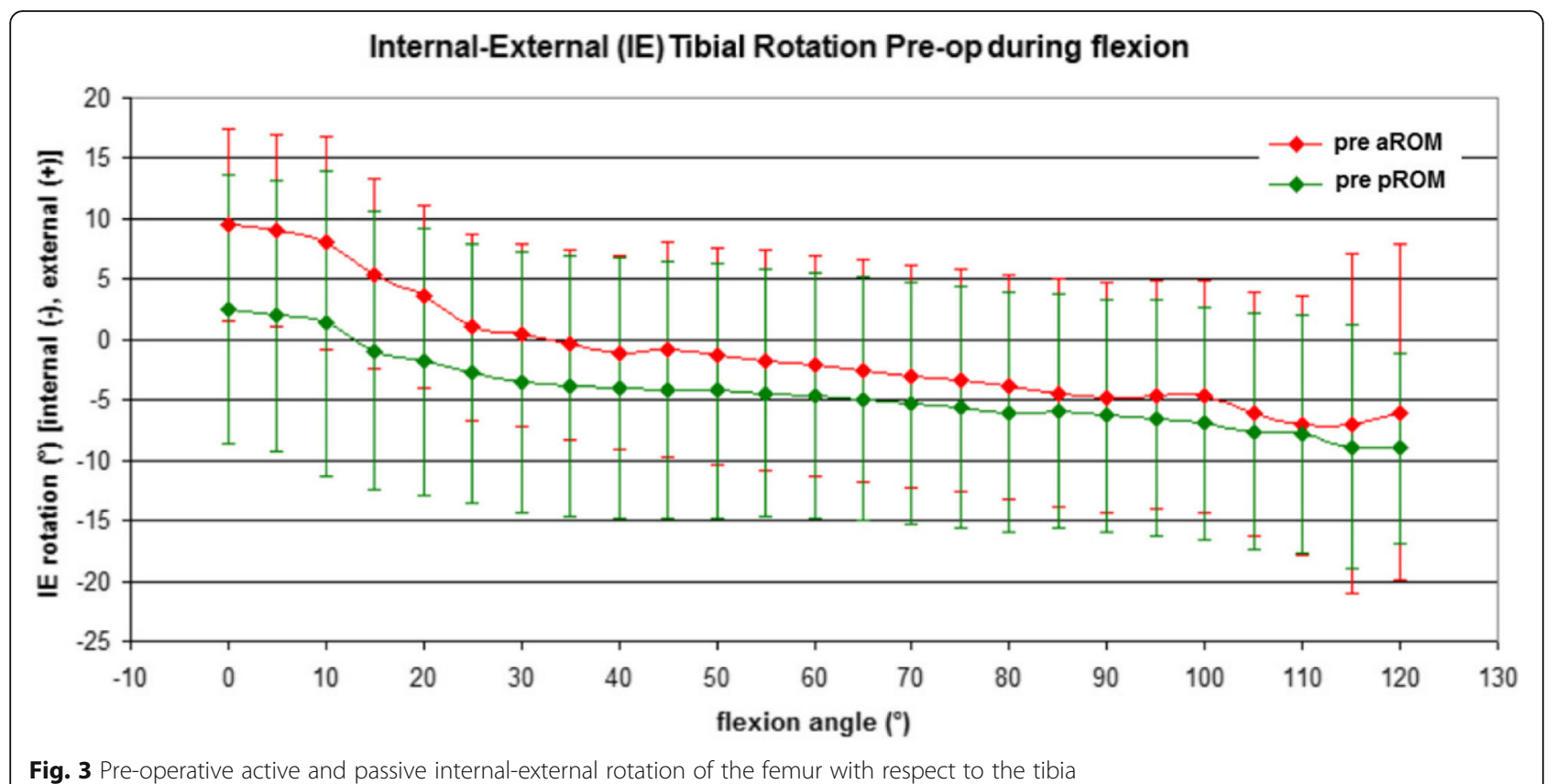

both aROM and pROM. No significant difference was found between aROM and pROM with paired Student t-test (n.s.).

Also, the post-operative rotation patterns were similar for active and passive motions (n.s.), showing a screwhome mechanism with an internal rotation of about $4^{\circ}$ for both curves (Fig. 4).

The post-operative IE rotation was reduced compared to pre-operative status for both aROM and pROM, however without statistical significance (n.s.).
Femoral AP translation during flexion

Pre-operative translation of the medial and lateral femoral condyles presented similar pattern in all conditions under study (Fig. 5, top).

The greater anterior translation was registered for lateral compartment, with a mean value of $63.5 \pm$ $20.8 \mathrm{~mm}$ for aROM and $52.2 \pm 26.9 \mathrm{~mm}$ for pROM. Differently, the medial compartment had a smaller mean displacement of $33.8 \pm 37.3 \mathrm{~mm}$ for aROM and

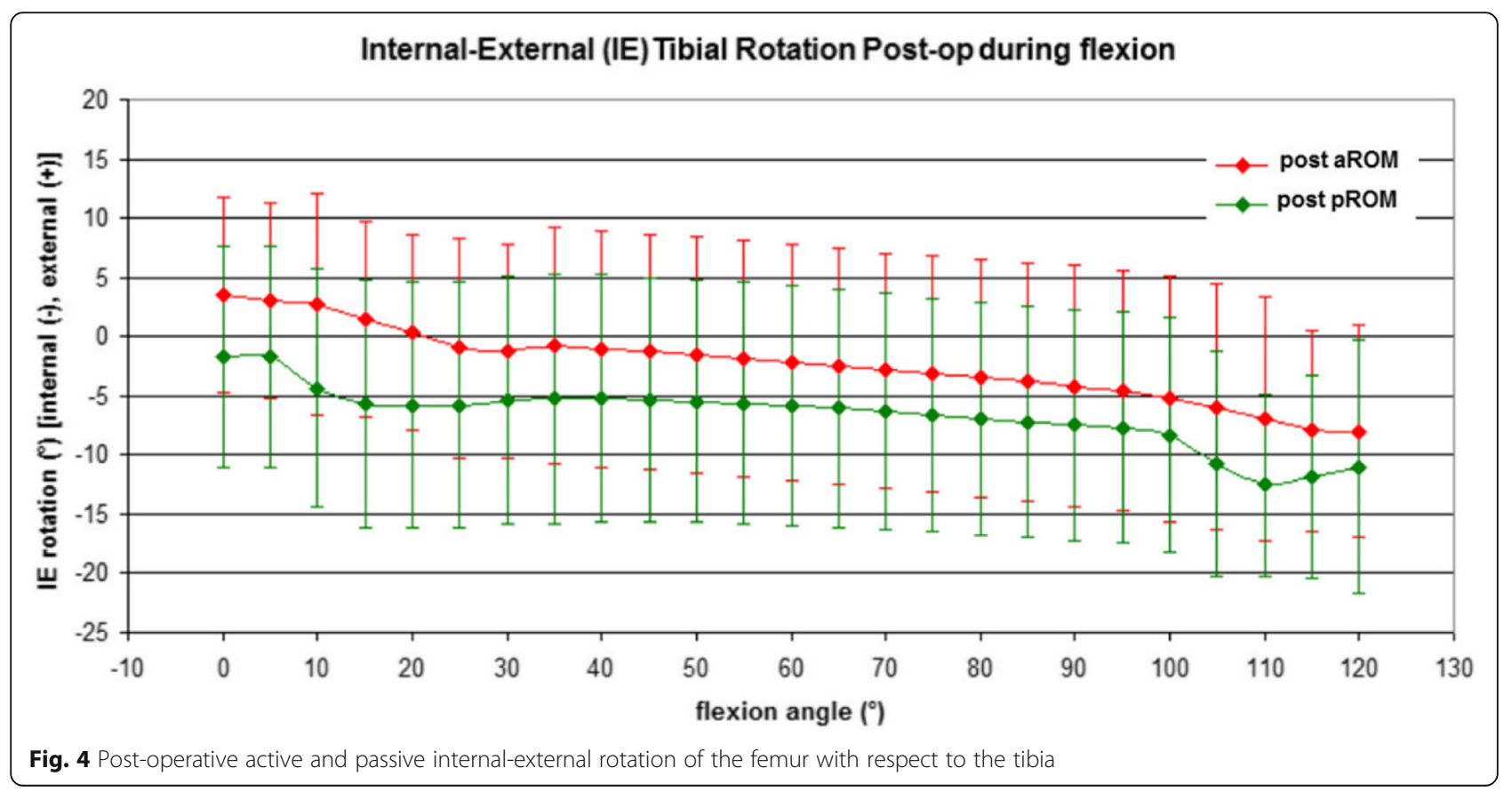




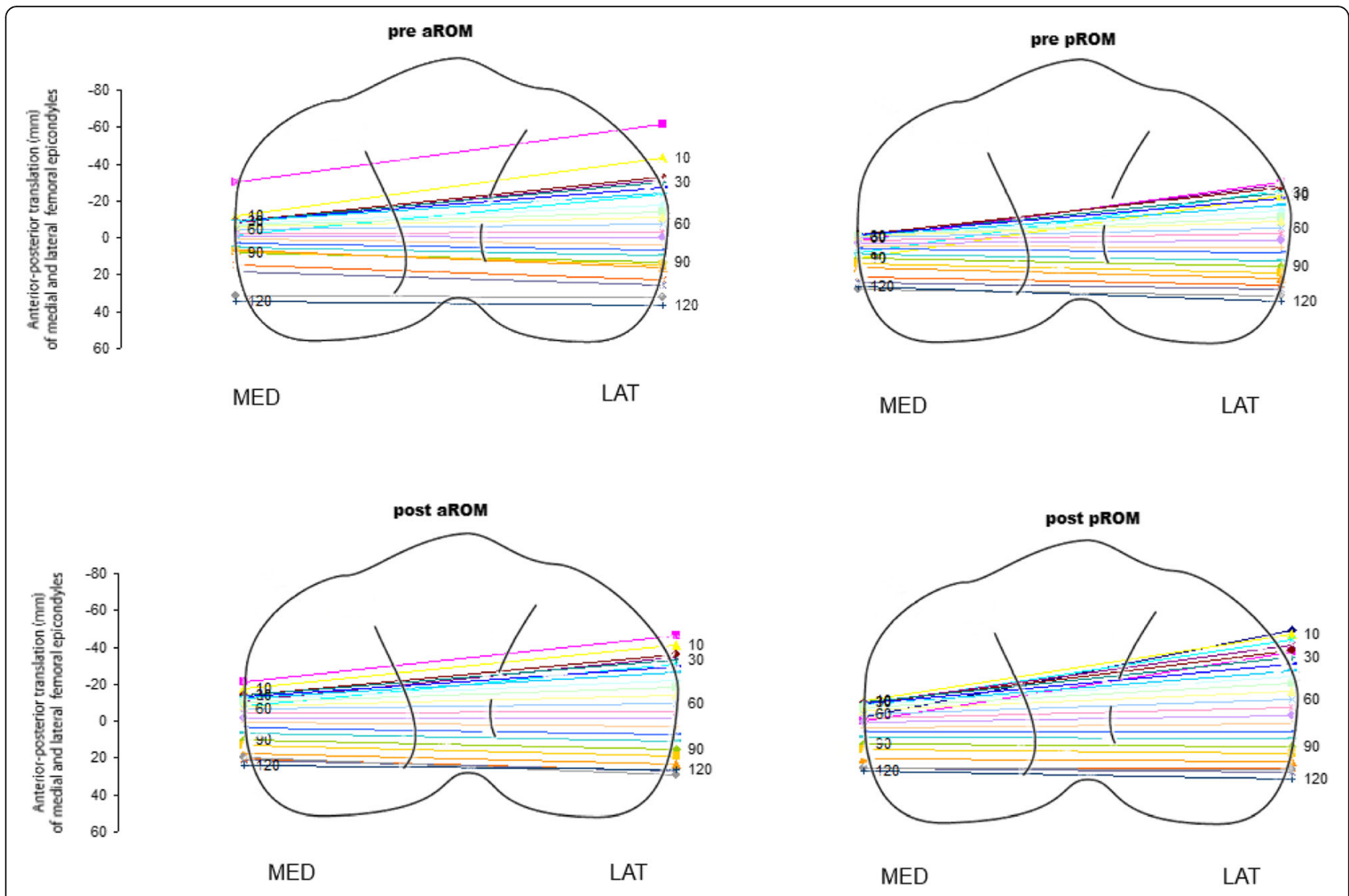

Fig. 5 Pre-operative (top) and post-operative (bottom) active and passive antero-posterior translation of the femur with respect to the tibia

$18.6 \pm 29.4 \mathrm{~mm}$ for pROM. For all patients, the translation occurred between $0^{\circ}$ and $90^{\circ}$ of flexion.

In the post-operative status (Fig. 5, bottom) after implant positioning, a slightly increased anterior displacement of the femur was found for lateral compartment during aROM $(72.6 \pm 29.4 \mathrm{~mm})$ and $\mathrm{pROM}(71.9 \pm 26.9$ $\mathrm{mm}$ ) with respect the pre-operative values. The displacement of medial compartment was $35.4 \pm 48.3 \mathrm{~mm}$ for aROM and $32.4 \pm 30.2 \mathrm{~mm}$ for pROM. No differences were registered between the pre-operative and postoperative status for both aROM and pROM $(p>0.05)$.

\section{Discussion}

The most important finding of the present study was that there are no significant differences between a flexion-extension movement actively performed by the patient and the same movement passively performed by the surgeon before and after TKA implantation.

The use of a navigation system in TKA has been proven to be extremely useful to perform a reliable intra-operative assessment of joint kinematics [7, 9-12, $15,18,19]$. However, so far, the presence or not of the muscle contraction still remained un-investigated, ignoring if it could affect intra-operative joint kinematics [6].
The main finding of the present study suggest that the passive ROM performed by the surgeon allows a reliable assessment of the knee kinematics; further, in this specific setup, muscle contraction do not significantly affect knee kinematics. Analyzing the available literature there was no study conducted in such scenario, and just few papers, to authors knowledge, compared post-operative aROM with post-operative pROM. Laidlaw et al. highlighted that the active ROM was significantly lower than passive ROM in a cohort of patients with a CR TKA design (aROM 100.3 degrees; pROM 115.2 degrees; $p<0.001$ ). The aROM was radiographically assessed at maximum flexion meanwhile the pROM clinically with a goniometer [13]. Song et al. compared passive maximum flexion without weight-bearing and other four flexion types, included active non-weight-bearing. They concluded that a greater maximum grade of knee flexion after TKA was achievable with the passive ROM with respect to the active ROM. However, this study was just focused on measuring the maximum degrees of flexion without any mention to the kinematical behavior during every task [20]. Although those results seem to be in contrast with the ones of the present study, it must be taken into account that substantially 
different methods were used, hence it is difficult to directly compare results and draw any solid conclusion.

Another notable finding of the present study was that TKA design restored the physiological screw home mechanism in early flexion $\left(0^{\circ}-30^{\circ}\right)$. The tibia performed an internal rotation of about $9^{\circ}$ during the active movement and $6^{\circ}$ during the passive one in pre-operative assessment, meanwhile after TKA implantation of $4^{\circ}$. Nevertheless, the comparison of pre and post-operative tasks seems to be in contrast with another kinematical study. Mooroka et al. [16] infact, analyzed pre and postoperative knee kinematic with CAS, finding out that before and after a Posterior Stabilized TKA implantation the physiological knee motion was not present.

An important aspect to consider is that the data showed in the present study had been acquired without weight bearing on the analyzed limb. Recently, instead, it has been demonstrated that significant differences in TKA kinematics occur during weight bearing respect to non-weight-bearing conditions. Bragonzoni et al., in fact, observed that in weight-bearing condition, the prosthesis femoral component had a significantly wider internal rotation during chair-raise instead of during an active ROM [3]. Therefore, it seems that more than muscle contraction is the weight bearing that affects the knee kinematic during a flexion-extension movement. This could be an explanation why in the present study no significant differences were found between pROM and aROM despite patient's muscle contraction.

The clinical relevance of the present study is that the pROM performed by the surgeon during surgery can accurately resemble the aROM with muscle contraction by the patient in open kinematic chain. This result comes to further support the use of CAS system in performing intra-operative analysis concerning knee biomechanics, thus, to provide fundamental information on both joint conditions and surgery procedures.

The findings of this study have to be seen in the light of some limitations. Both aROM and pROM data were acquired after joint capsule were opened, so it could have possibly altered the kinematical behavior of the knee, due to the loss of its role of joint restrainer. However, this condition was present in both evaluations and therefore it is possible that this factor did not affected too much the primary endpoint. The presence of the tourniquet could have affected the kinematical evaluation in particular the aROM acquisition. The postoperative data presented refer to a specific prothesis design, hence other TKA designs could give different results. The intra-operative assessment of the ROM still remains an empirical evaluation of the knee movement which, for sure, resemble the daily living movement but do not completely investigate the knee motion in all his complexity. Finally, despite the power analysis indicated a minimum sample size of 24 subjects with a larger cohort the differences detected could become statistically significant even though the clinical significance has to be defined.

Despite these limitations, to the best knowledge of the authors this represents the first study comparing active ROM performed by the patients intra operatively and the same movement performed by the surgeon and poses the basis for further studies that compares intraoperative kinematic with active daily life motor tasks in order to increase the knowledge on knee biomechanics.

\section{Conclusion}

No significant differences between a flexion-extension movement actively performed by the patient and the same movement passively performed by the surgeon before and after TKA implantation were detected during an intra-operative kinematic evaluation using a navigation system. This result comes to further support the accuracy of the CAS system in intra-operative knee kinematic evaluations without the need for patient active contraction which seems to not affect the knee kinematics in the described setting.

\section{Abbreviations}

ACL: Anterior Cruciate Ligament; AP: Anterior/Posterior; aROM: Active Range of Motion; CAS: Computer-Assisted-Surgery; CR: Cruciate Retaining; CSE: Combined Spinal and Epidural; IE: Internal/External; JCS: joint coordinate reference system; MB: Mobile Bearing; ML: Medial/Lateral; OA: osteoarthritis; PD: Proximal/Distal; pROM: Passive Range of Motion; TEA: trans-epicondylar axis; TKA: Total Knee Arthroplasty; WSL: Whiteside Line

\section{Acknowledgements}

Not applicable.

\section{Authors' contributions}

A.G.: design of the study, data interpretation, writing manuscript. N.P.: data interpretation, writing manuscript, correspondence. N.F.L.: design of the study, data processing, writing manuscript. M.M.: design of the study, surgical procedure, writing manuscript. M.C.: design of the study, surgical procedure, writing manuscript. G.M.M.M.: data interpretation, writing manuscript. F.C.: design of the study, data processing, writing manuscript. S.Z.: design of the study, surgical procedure, writing manuscript. all authors read and approved the manuscript.

\section{Funding}

The authors declare that no funding has been used for the present study.

Availability of data and materials

All data generated or analysed during this study are included in this published article.

Ethics approval and consent to participate

The study was approved by the ethical committee of the IRCCS Rizzoli Orthopedic Institute (protocol number 11551/CE/US/ml, 5 May 2006).

Consent for publication

Not applicable.

Competing interests

The authors declare that they have no competing interests. 


\section{Author details}

'Il Clinica Ortopedica e Traumatologica, IRCCS, Istituto Ortopedico Rizzoli, Bologna, Italy. ${ }^{2}$ Dipartimento Scienze Biomediche e Neuromotorie-DIBINEM, Università di Bologna, Bologna, Italy. ${ }^{3}$ Dipartimento di Ingegneria dell'Informazione, Università degli Studi di Brescia, via Branze, 3825123 Brescia, BS, Italy. ${ }^{4}$ Humanitas Clinical and Research Center, Milan, Italy. 5 Laboratorio di Biomeccanica, Istituto Ortopedico Rizzoli, via di Barbiano 1/ 10, I-40136 Bologna, BO, Italy.

Received: 6 January 2020 Accepted: 27 February 2020

Published online: 12 March 2020

\section{References}

1. Akkawi I, Colle F, Bruni D, Raspugli GF, Bignozzi S, Zaffagnini S, lacono F Marcacci M (2015) Deep-dished highly congruent tibial insert in CR-TKA does not prevent patellar tendon angle increase and patellar anterior translation. Knee Surg Sports Traumatol Arthrosc 23:1622-1630

2. Bignozzi S, Zaffagnini S, Akkawi I, Marko T, Bruni D, Neri MP, Colle F, Marcacci M (2014) Three different cruciate-sacrificing TKA designs: minor intraoperative kinematic differences and negligible clinical differences. Knee Surg Sports Traumatol Arthrosc 22:3113-3120

3. Bragonzoni L, Marcheggiani Muccioli GM, Bontempi M, Roberti di Sarsina T, Cardinale U, Alesi D, Acono F, Neri MP, Zaffagnini S (2019) New design total knee arthroplasty shows medial pivoting movement under weight-bearing conditions. Knee Surg Sports Traumatol Arthrosc 27:1049-1056

4. Casino D, Martelli S, Zaffagnini S, Lopomo N, lacono F, Bignozzi S, Visani A Marcacci M (2009) Knee stability before and after total and unicondylar knee replacement: In vivo kinematic evaluation utilizing navigation. J Orthop Res 27(2):202-207

5. Cole GK, Nigg BM, Ronsky JL, Yeadon MR (1993) Application of the joint coordinate system to three-dimensional joint attitude and movement representation: a standardization proposal. J Biomech Eng 115:344-349

6. Doro LC, Hughes RE, Miller JD, Schultz KF, Hallstrom B, Urquhart AG (2008) The reproducibility of a kinematically-derived axis of the knee versus digitized anatomical landmarks using a knee navigation system. Open Biomed Eng J 2:52-56

7. Fritzsche H, Beyer F, Postler A, Lützner J (2018) Different intraoperative kinematics, stability, and range of motion between cruciate-substituting ultracongruent and posterior-stabilized total knee arthroplasty. Knee Surg Sports Traumatol Arthrosc 26:1465-1470

8. Grood ES, Suntay WJ (1983) A joint coordinate system for the clinical description of three dimensional motions: application to the knee. J Biomech Eng 105:136-144

9. Ishida K, Shibanuma N, Matsumoto T, Sasaki H, Takayama K, Matsuzaki T, Tei K, Kuroda R, Kurosaka M (2016) Navigation-based femorotibial rotation pattern correlated with flexion angle after total knee arthroplasty. Knee Surg Sports Traumatol Arthrosc 24:89-95

10. Ishida K, Shibanuma N, Matsumoto T, Sasaki H, Takayama K, Toda A, Kuroda $R$, Kurosaka M (2015) Factors affecting intraoperative kinematic patterns and flexion angles in navigated total knee arthroplasty. Knee Surg Sports Traumatol Arthrosc 23:1741-1747

11. Kim TW, Lee SM, Seong SC, Lee S, Jang J, Lee MC (2016) Different intraoperative kinematics with comparable clinical outcomes of ultracongruent and posterior stabilized mobile-bearing total knee arthroplasty. Knee Surg Sports Traumatol Arthrosc 24:3036-3043

12. Klein GR, Parvizi J, Rapuri VR, Austin MS, Hozack WJ (2004) The effect of tibial polyethylene insert design on range of motion: evaluation of in vivo knee kinematics by a computerized navigation system during total knee arthroplasty. J Arthroplast 19:986-991

13. Laidlaw MS, Rolston LR, Bozic KJ, Ries MD (2010) Assessment of tibiofemoral position in total knee arthroplasty using the active flexion lateral radiograph. Knee 17:38-42

14. Martelli S, Zaffagnini S, Bignozzi S, Bontempi M, Marcacci M (2006) Validation of a new protocol for computer-assisted evaluation of kinematics of double-bundle $\mathrm{ACL}$ reconstruction. Clin Biomech (Bristol, Avon) 21:279-287

15. Matsumoto T, Takayama K, Muratsu H, Matsushita T, Kuroda R, Kurosaka M (2015) Semimembranosus release reduces Tibial internal rotation and flexion angle in cruciate-retaining Total knee Arthroplasty. J Arthroplast 30: $1537-1541$
16. Morooka T, Okuno M, Seino D, Iseki T, Fukunishi S, Kobashi S, Yoshiya S (2018) Intraoperative kinematic analysis of posterior stabilized total knee arthroplasty with asymmetric helical post-cam design. Eur J Orthop Surg Traumatol. https://doi.org/10.1007/s00590-018-2318-4

17. Pickering AE, Parry MG, Ousta B, Fernando R (1999) Effect of combined spinal-epidural ambulatory labor analgesia on balance. Anesthes 91:436-441

18. Seito N, Onodera T, Kasahara Y, Kondo E, Iwasaki N, Majima T (2017) Preoperative knee deformity and kinematics impact postoperative knee kinematics in total knee arthroplasty. Knee. https://doi.org/10.1016/j.knee. 2017.08.056

19. Seon JK, Park JK, Jeong MS, Jung WB, Park KS, Yoon TR, Song EK (2011) Correlation between preoperative and postoperative knee kinematics in total knee arthroplasty using cruciate retaining designs. Int Orthop 35: 515-520

20. Song YD, Jain N, Kang YG, Kim TY, Kim TK (2016) Active flexion in weight bearing better correlates with functional outcomes of Total knee Arthroplasty than passive flexion. Knee Surg Relat Res 28:118-129

21. Wiles AD, Thompson DG, Frantz DD (2004) Accuracy assessment and interpretation for optical tracking systems. Medical Imaging 2004: Visualization. Image-Guided Procedures, and Display International Society for Optics and Photonics. https://doi.org/10.1117/12.536128

\section{Publisher's Note}

Springer Nature remains neutral with regard to jurisdictional claims in published maps and institutional affiliations.

\section{Submit your manuscript to a SpringerOpen ${ }^{\circ}$ journal and benefit from:}

- Convenient online submission

- Rigorous peer review

- Open access: articles freely available online

High visibility within the field

- Retaining the copyright to your article

Submit your next manuscript at $\boldsymbol{\nabla}$ springeropen.com 